\title{
Student Science Research in Education Abroad
}

Study abroad provides many opportunities for students. My own college's goals for international programs state that "students are expected to gain new perspectives on their fields of study, develop intercultural competencies and communication skills, learn others' perspectives, and reflect on their own assumptions and values while abroad" (Beloit College Office of International Education, 2005). Research abroad by students ideally provides these same benefits, better preparing them to function in their chosen fields. The views expressed in this essay come from my perspective as a biology and geology professor who has had the opportunity to travel and do research in a number of different and diverse countries. In addition, they stem from conversations with liberal-arts-college students, and with Sarah Davidson. Three aspects of research abroad are pertinent to this discussion: different approaches to science research, the need to have global coverage in our studies, and the site-specific nature of many scientific studies.

\section{A p proaches to Science}

There are two approaches to doing science: basic and applied. The passion to learn about the natural world drives basic (or fundamental) research, whereas economics or the desire to improve life for humans guides applied work. During WWII and since, the United States developed an appreciation of how basic research can lead to economically profitable applications, many of which bettered our living conditions (e.g., solutions to health and sanitation problems). Since WWII government began funding basic research in earnest while industry primarily funded applied work. In practice, however, our approach to doing science research often blends aspects of basic and applied research, and new national policy reflects the diverse types of research we do (House Committee on Science, 1998).

At the undergraduate level, and particularly perhaps at liberal arts colleges, we stress the love of learning about the natural world (i.e., basic) or improving the human condition (applied); we do not stress the immediate economic value of the research. Such is not the case in many countries, many of which do not have the financial means for funding basic research. Thus, scientists from these countries take a very different approach to science. I offer 
two examples, one personal and one from a student, to make my point. In 1990 during a visit to Georgia (at that time a part of the Soviet Union), I was discussing wetlands with a Georgian colleague. I could not understand her points, a fact that I initially attributed to language difficulties, though my colleague seemed to have an excellent facility with English. I finally realized the source of confusion. My approach to the problem reflected my environmental background_-"save the wetlands!"- — whereas hers represented her economic bias_-"drain the wetlands!" We were talking past each other because of our different approaches to the problem.

Sarah had a similar experience working with Mongolian students in summer of 2003. Unless the Mongolian students could see a direct economic application to doing the field study, they saw no value in doing it. They wanted to tackle research that would better prepare them for a job in economic geology, whereas Sarah most wanted to reconstruct a small piece of geologic history.

Increasingly, we in the United States will be involved in international collaborations. Research in an international setting will help prepare students by making them aware of the potential for different approaches, thus facilitating communication-communication that goes beyond the level of language differences. Such experiences also inevitably lead one to think about why there are differences in approach, which provides insight into the history of the peoples and their culture.

\section{ReIatively UnexpIored Areas}

Because of different approaches to science, philosophies about science, ability to fund research, and probably many other reasons, our global understanding of the natural world is incomplete. For example, Davidson's research (Davidson, this volume) characterized the source of particles that make up sedimentary rocks in an area of the Gobi Desert and unraveled the history of sedimentation. Little work has been done in this region because of its remoteness. Sarah's work in Mongolia contributes a piece to understanding the geologic history of the region. Many of our models for understanding earth history are based on information gathered from areas where Europeans or North Americans have worked extensively; the reconstructed history, therefore, is biased. Large parts of Asia are not represented well in the models. Only over the last few decades have we recognized the importance of this void, and have developed working relationships with people of these areas, in part facilitated by changes in political philosophy in many Asian countries. A similar void existed in the reconstruction of the history of life. A couple of decades ago, 
after Deng Xiaoping succeeded Mao Zadong in China, North American and European paleontologists began collaborating with Chinese paleontologists, and numerous and incredible finds of dinosaurs, bird-dinosaur links, and earliest life from China were reported (e.g., see Stokstad, 2001). Now, Chinese paleontologists publish regularly in mainstream English journals and their studies force paleontologists to revise earlier models. It might be that the increase in publications would have occurred solely due to the change in the political regime and attitude toward developing the economy, but the increasing number of international collaborations suggests the importance of collaborative works throughout these past 26 years. Similar changes and revelations have been slower to take place in the field of sedimentology. More work like Sarah's will highlight the scientific importance of these less studied geographic areas, and should support more collaborative work in the future.

\section{Site-Specific Studies}

There's only so much you can learn in the classroom or even in a field lab because some work is site dependent. This is particularly true in areas dealing with ecology and health. For example, in the Upper Midwest, we can lecture about tropical rain forests and attempt to give the student a sense of the ecosystem by visiting a local oak and maple woodland. It's not the same as being in the Amazon rain forest where the trees, soils, animals, and smells and sounds are all different. Working in a different ecosystem promotes an understanding not typically acquired through book study. Ideally, it instills a sense of ownership and desire to protect, and also brings an awareness of non-related conditions. For example, the need for malaria medications heightens the awareness that health issues are different in tropical environments.

In conclusion, natural science research abroad helps students understand different approaches to science so that they can better communicate with other scientists. It allows them to study ecosystems, health-related issues, or other natural resources that are not available in the United States. The heightened international communication also helps to build a less-biased vision of the natural world. Ideally, the research leads to numerous questions and investigations that may not directly relate to the research, but may enhance understanding and respect for others, which may help in tackling local and global issues in the future.

\section{C a r o I M a n ki e w i c z}

\section{Professor of Biology and Geology}

Beloit College 